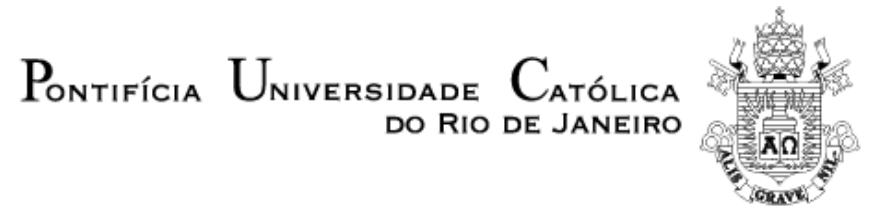

Victor Kraemer Wermelinger Sancho Araujo

\title{
Avaliação de custos para a produção de biodiesel a partir de óleos residuais fritura
}

Dissertação apresentada como requisito parcial para obtenção do título de Mestre pelo Programa de Pós-Graduação em Engenharia Industrial da PUC-Rio.

Orientador: Prof. Sílvio Hamacher

Rio de Janeiro

Abril de 2008 


$$
\begin{array}{r}
\text { Pontifícia } U_{\text {niversidade }} \text { Católica }_{\text {Do Rio de Janeiro }} \\
.
\end{array}
$$

\section{Victor Kraemer Wermelinger Sancho Araujo}

\section{Avaliação de custos para a produção de biodiesel a partir de óleos residuais fritura}

Prof. Sílvio Hamacher Orientador Departamento de Engenharia Industrial - PUC-Rio

Prof. Luiz Felipe Roris Rodriguez Scavarda do Carmo Departamento de Engenharia Industrial - PUC-Rio Dr. Eduardo Homem de Siqueira Cavalcanti INT/SECTI-RJ

Prof. José Eugenio Leal Coordenador Setorial do Centro Técnico Científico - PUC-Rio 
Todos os direitos reservados. É proibida a reprodução total ou parcial do trabalho sem autorização da universidade, da autora e do orientador.

\section{Victor Kraemer Wermelinger Sancho Araujo}

Graduou-se em Engenharia de Produção pela PUC-Rio em 2005. Durante a graduação, estagiou em empresa do ramo de telecomunicações, atuando nas áreas de Previsão de Indicadores, e completou o curso seqüencial em empreendedorismo da PUC-Rio. Em 2006, ingressou no Programa de Pós-Graduação em Engenharia Industrial da PUC-Rio para obtenção do título de Mestre. Nesse período, trabalhou como pesquisador no Projeto Biodiesel.

Ficha Catalográfica

Araujo, Victor Kraemer Wermelinger Sancho

Avaliação de custos para a produção de biodiesel a partir de óleos residuais fritura / Victor Kraemer Wermelinger Sancho Araujo ; orientador: Silvio Hamacher. $-2008$

97 f. : II. ; $30 \mathrm{~cm}$

Dissertação (Mestrado em Engenharia Industrial)Pontifícia Universidade Católica do Rio de Janeiro, Rio de Janeiro, 2008.

Inclui bibliografia

1. Engenharia industrial - Teses. 2. Biodiesel. 3. Problema de Roteamento de veículos. 4. Óleo Residual de fritura. 5. Rio de Janeiro. 6. Logística. I. Hamacher, Sílvio. II. Pontifícia Universidade Católica do Rio de Janeiro. Departamento de Engenharia Industrial. III. Título. 


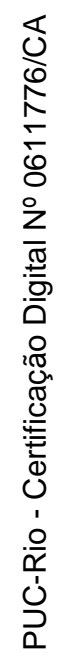

A Deus 


\section{Agradecimentos}

Agradeço, antes de tudo, a Deus, por me dar forças nos momentos difíceis e por me permitir atingir este estágio. Tudo o que alcancei e o que hei de alcançar por Ele me foi e será permitido.

À minha família, pelo suporte e pelas críticas. Certamente devo a ela muitos de meus valores e conquistas.

Ao meu orientador, professor Silvio Hamacher, pela paciência, disponibilidade e inteligência na transmissão de seus conhecimentos.

Aos meus amigos, com destaque aos grandes amigos verdadeiros da época de colégio e aos amigos do Effatá, pelo incentivo. Aos amigos de graduação da PUC e aos meus colegas de mestrado, pelos momentos felizes e partilhas de estudo. Em especial, agradeço à Patrícia, pelo companheirismo, pelos momentos de descontração, pelas conversas e pela crença no meu potencial.

Aos funcionários e professores, que, de alguma forma, me transmitiram conhecimentos, educação e disciplina para toda vida. Sou especialmente grato aos professores Luiz Felipe Scavarda, Madiagne Diallo e José Eugênio Leal, pela amizade e confiança no meu trabalho.

Ao CNPq e à Fundação de Apoio à Pesquisa da Bahia (FAPESB-BA), pelo apoio financeiro, e a Eduardo Cavalcanti, coordenador do Programa RioBiodiesel.

A todos aqueles que, de alguma forma, contribuíram para este trabalho. 


\section{Resumo}

Araujo, Victor Kraemer Wermelinger Sancho. Hamacher, Silvio. Avaliação de custos para a produção de biodiesel a partir de óleos residuais fritura. Rio de Janeiro, 2008. 97 p. Dissertação de Mestrado Departamento de Engenharia Industrial, Pontifícia Universidade Católica do Rio de Janeiro.

A busca pelo desenvolvimento sustentável tem como importante fator diferencial as fontes de energia renováveis. O biodiesel desponta como uma das alternativas mais relevantes, mas suas formas de obtenção no Rio de Janeiro não foram suficientemente investigadas. Este trabalho identifica a oportunidade da produção de biodiesel a partir de óleos residuais de fritura neste cenário, enfatizando os custos de transporte do óleo desde os principais produtores comerciais até a obtenção do biocombustível. O objetivo é avaliar os custos de forma a verificar a viabilidade do emprego desta alternativa. Para tanto, foram estudadas as diversas ferramentas de resolução do Problema de Roteamento de Veículos e foi proposto um algoritmo que visa à otimização dos custos. A formulação matemática utilizada baseia-se numa extensão de algoritmos clássicos, como o apresentado por Arenales et al. (2007), e nas equações desenvolvidas em Kallehauge (2006). Os resultados do modelo de roteamento, atrelados aos custos de produção, impostos e insumos, foram comparados com informações sobre a comercialização do biodiesel, comprovando sua viabilidade econômica. A consolidação dos dados obtidos aponta a produção de biodiesel a partir de óleo residual de fritura como viável, com custos logísticos equivalentes a $\mathrm{R} \$ 0,19$ por litro e custo final de $\mathrm{R} \$ 1,22$ por litro.

\section{Palavras-chave}

Biodiesel; Problema de Roteamento de Veículos; Óleo Residual de Fritura; Rio de Janeiro; Logística. 


\section{Abstract}

Araujo, Victor Kraemer Wermelinger Sancho. Hamacher, Silvio. Cost evaluation for biodiesel production from waste cooking oil. Rio de Janeiro, 2008. 97 p. M. Sc. Dissertation - Departamento de Engenharia Industrial, Pontifícia Universidade Católica do Rio de Janeiro.

The search for a sustainable development has in renewable energy sources an important differential factor. Biodiesel is one of the most important alternatives, but its obtainment forms in Rio de Janeiro have not been investigated enough. This work identifies the opportunity of biodiesel production from waste cooking oil in this scenery, emphasizing oil's transport costs until factories, where it is possible to obtain biodiesel in its final form. The objective is to evaluate costs in order to verify viability of this alternative source of energy. Hence, this research analysed several tools for solving Vehicle Routing Problem and it proposes an algorithm that results in cost optimization. The adapted mathematic formulation is based in an extension of classic algorithms, like those presented by Arenales (2007), and in equations developed by Kallehauge (2006). The routing model results, linked to production, tributes and input costs, have been compared with information about biodiesel commercialization, verifying its economic viability. The data consolidation obtained indicates that the biodiesel production from waste cooking oil is viable, with logistic costs equal to $\mathrm{R} \$ 0,19$ per liter and final cost equal to $\mathrm{R} \$ 1,22$ per liter.

\section{Key words}

Biodiesel; Vehicle Routing Problem; Waste Cooking Oil; Rio de Janeiro; Logistics 


\section{Sumário}

1 INTRODUÇÃO

1.1. OBJETIVOS 15

1.2. HIPÓTESES E SUPOSIÇÕES

1.3. DELIMITAÇÃO DO ESTUDO 15

1.4. MOTIVAÇÃO 16

1.5. RELEVÂNCIA 16

1.6. ESTRUTURA 17

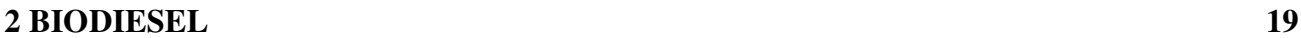

2.1. IMPORTÂNCIA DO BIODIESEL 19

2.2. DEFINIÇÕES 21

2.3. PROCESSOS DE TRANSFORMAÇÃO 22

2.4. MERCADO DO BIODIESEL 26

2.5. A CADEIA PRODUTIVA DO BIODIESEL 26

2.5.1. Comparação entre matérias-primas 29

2.6. BIODIESEL A PARTIR DE RESÍDUOS 31

2.7. LEILÕES DE BIODIESEL 33

3 SISTEMAS DE ROTEAMENTO

3.1. O PROBLEMA DO CAIXEIRO VIAJANTE (PCV) 38

3.2. O PROBLEMA DO CARTEIRO CHINÊS 42

3.3. PROBLEMA DE ROTEAMENTO DE VEÍCULOS

4 METODOLOGIA $\quad 47$

4.1. TIPO DE PESQUISA

4.2. UNIVERSO E AMOSTRA

4.3. COLETA DE DADOS 49

4.4. TRATAMENTO DOS DADOS 50

4.4.1. Google Earth 50

4.4.2. AIMMS 51

4.5. LIMITAÇÕES DO MÉTODO 52

4.6. CRONOGRAMA 53

5 MATERIAIS E MÉTODOS

5.1. TESTES $\quad 60$

6 ANÁLISE DE RESULTADOS

6.1. CUSTOS LOGÍSTICOS 64

6.2. CUSTOS DE PRODUÇÃO 73

6.3. CUSTOS NÃO OPERACIONAIS 75 
6.3.1. Tributos 75

6.3.2. Aquisição de Insumos

6.4. FORMAÇÃO DOS CUSTOS DO BIODIESEL 79

6.5. ANÁLISE COMPARATIVA 80

7 CONCLUSÕES $\quad 82$

7.1. SUGESTÕES PARA TRABALHOS FUTUROS 83

$\begin{array}{lr}\text { REFERÊNCIAS BIBLIOGRÁFICAS } & 84\end{array}$

APÊNDICE I: ESPECIFICAÇÕES $\quad 88$

PROPRIEDADES FÍSICAS E QUÍMICAS

APÊNDICE II: PROCESSOS DE TRANSFORMAÇÃO DO BIODIESEL 91

CRAQUEAMENTO 92

ESTERIFICAÇÃO 92

APÊNDICE III: QUESTIONÁRIO

APÊNDICE IV: HORÁRIOS DE CHEGADA 96 


\section{Lista de Figuras}

Figura 1: Reação de transesterificação por rota metílica $\quad 23$

Figura 2: Obtenção do biodiesel através do processo de transesterificação 24

Figura 3: Transesterificação considerando tratamento do álcool e glicerina 24

Figura 4: Fluxograma das cadeias produtivas no Brasil $\quad 27$

Figura 5: Distribuição dos custos de produção do biodiesel por matéria-prima 29

Figura 6: Decomposição dos custos variáveis do biocombustível 30

Figura 7: Biodiesel proveniente do óleo de fritura

Figura 8: Fluxograma da produção de biodiesel a partir de óleos residuais 32

Figura 9: Jogo de Hamilton $\quad 38$

Figura 10: Desenho da cidade de Königsberg $\quad 42$

Figura 11: Conjunto de rotas para o PRV 46

Figura 12: Consumo de óleo de fritura e número de clientes nos diferentes $\begin{array}{ll}\text { estabelecimentos } & 48\end{array}$

Figura 13: Grafos das variações no roteamento de veículos

Figura 14: Representação dos grupamentos para roteamento no mapa do Rio de Janeiro 66

Figura 15: Rotas obtidas para o terceiro cluster $\quad 70$

Figura 16: Novas rotas obtidas para o terceiro cluster $\quad 72$

Figura 17: Destino final dos óleos residuais de fritura nas lanchonetes pesquisadas $\quad 77$

$\begin{array}{ll}\text { Figura 18: Série histórica de preços do metanol } & 78\end{array}$

Figura 19: Comparação de custos para produção de biodiesel obtidos pelos $\begin{array}{ll}\text { diferentes trabalhos e leilão } & \mathbf{8 1}\end{array}$

Figura 20: Reação de esterificação $\quad 93$ 


\section{Lista de Tabelas}

Tabela 1: Definição de biodiesel $\quad 22$

Tabela 2: Comparação das rotas metílica e etílica $\quad 25$

Tabela 3: Classificação do óleo residual de acordo com sua origem 32

Tabela 4: Propriedades da transesterificação supercrítica e convencional 33

Tabela 5: Volume de biodiesel arrematado por empresa $\left(\mathbf{m}^{3}\right) \quad 34$

Tabela 6: Quadro resumo de dados dos leilões

Tabela 7: Matriz O/D utilizada para testes iniciais $\quad 60$

Tabela 8: Valores de demanda utilizados para o caso inicial $\quad 61$

Tabela 9: Horas de chegada do veículo em cada cliente

Tabela 10: Custos dos veículos $\quad 63$

Tabela 11: Variação de rotas de acordo com as capacidades dos veículos $\quad 63$

Tabela 12: Configuração das rotas para os diferentes clusters $\quad 67$

Tabela 13: Dados relativos ao processamento do algoritmo no AIMMS

Tabela 14: Horários de chegada para atendimento dos clientes no terceiro cluster $\quad 70$

Tabela 15: Configuração de rotas clusters após mudança de veículo

Tabela 16: Horários de chegada para atendimento dos clientes no terceiro cluster após mudança de veículo $\quad 72$

$\begin{array}{ll}\text { Tabela 17: Investimento e custos operacionais para biodiesel } & 74\end{array}$

Tabela 18: Tributos incidentes na produção de biodiesel $\quad 76$

Tabela 19: Coeficientes técnicos para produção de biodiesel $\quad 77$

$\begin{array}{lc}\text { Tabela 20: Formação do preço do biodiesel } & 80\end{array}$

$\begin{array}{lr}\text { Tabela 21: Especificações do biodiesel } & 88\end{array}$

Tabela 22: Propriedades físico-químicas do biodiesel $\quad 89$

Tabela 23: Descrição das etapas da transesterificação 91

Tabela 24: Horários de chegada para atendimento dos clientes na Barra 96

Tabela 25: Horários de chegada para atendimento dos clientes na Zona Sul 96

Tabela 26: Horários de chegada para atendimento dos clientes na Barra após mudança de $\begin{array}{ll}\text { veículo } & 96\end{array}$

Tabela 27: Horários de chegada para atendimento dos clientes na Zona Sul após mudança de veículo 